\title{
Cuidado a la persona con pluripatología: revisión integrativa
}

\section{Care for the person with pluripatology: integrative review}

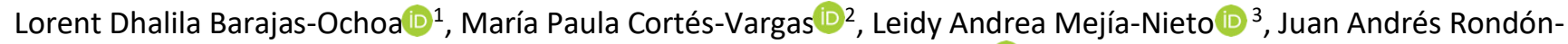 \\ Ochoa (1D ${ }^{4}$, Sonia Carreño-Moreno (iD) 5
}

1. Universidad Nacional de Colombia. Bogotá, Colombia. Correo: Idbarajaso@unal.edu.co - https://orcid.org/0000-0001-6956-1525

2. Universidad Nacional de Colombia. Bogotá, Colombia. Correo: mpcortesv@unal.edu.co - https://orcid.org/0000-0002-6077-1849

3. Universidad Nacional de Colombia. Bogotá, Colombia. Correo: lamejian@unal.edu.co - https://orcid.org/0000-0002-2297-6624

4. Universidad Nacional de Colombia. Bogotá, Colombia. Correo: jarondono@unal.edu.co - https://orcid.org/0000-0001-6651-9102

5. Universidad Nacional de Colombia. Bogotá, Colombia. Correo: spcarrenom@unal.edu.co - https://orcid.org/0000-0002-4386-6053

Tipología: Artículo de revisión

Para citar este artículo: Barajas-Ochoa LD, Cortés-Vargas MP, Mejía-Nieto LA, Rondón-Ochoa JA, Carreño-Moreno S. Cuidado a la persona con pluripatología: revisión integrativa. Duazary. 2021 abril; 18(2): 213-224. Doi: https://doi.org/10.21676/2389783X.4075

\section{RESUMEN}

Palabras clave: multimorbilidad; atención integral de salud; enfermería basada en la evidencia; comorbilidad; revisión.
El objetivo de este artículo es conocer e integrar los hallazgos derivados de la producción científica disponible respecto a las experiencias y necesidades de cuidado de las personas con pluripatología. Se realizó una revisión integrativa descriptiva, en la que se hizo una búsqueda en bases de datos limitando entre los años 2009-2019 y utilizando términos MeSH y DeCS como "multimorbidity", "comorbidity", "health care" y "nursing care", seleccionados bajo los criterios de rigor de la investigación cualitativa y cuantitativa, para finalmente analizar a profundidad 25 artículos originales. Los hallazgos se agruparon en cuatro categorías temáticas: necesidades de cuidado en la pluripatología, sentimientos ante la vivencia, barreras de atención en salud, y sistemas de apoyo. El mayor nivel de evidencia y de número de estudios se presenta en el tema de necesidades de cuidado. Se concluye que la asistencia brindada a las personas con pluripatología requiere ser reestructurada teniendo en cuenta las necesidades específicas que surgen en ellas, buscando fortalecer la autoestima, la autonomía, la capacidad de afrontamiento y la autogestión de la salud.

\section{ABSTRACT}

\section{Keywords:} Multimorbidity ;

Comprehensiv e health care; Evidencebased nursing; Comorbidity; Review.
The objective of this article is to know and integrate the findings derived from the available scientific production regarding the experiences and care needs of people with multiple pathologies. A descriptive integrative review was carried out, in which a search was made in databases limiting between the years 2009 - 2019, using MeSH and DeCS terms such as Multimorbidity, Comorbidity, Health Care and Nursing care, in which they were selected under the criteria rigor of qualitative and quantitative research, to finally analyze in depth 25 original articles. The findings were grouped into four thematic categories, which were Care Needs in Multiple Pathology, Feelings of Experience, Health Care Barriers, and Support Systems. The highest level of evidence and number of studies is presented in the topic of Needs for care. It is concluded that the assistance provided to people with multiple pathologies needs to be restructured taking into account the specific needs that arise in them, seeking to strengthen self-esteem, autonomy, coping capacity and self-management of health. 


\section{INTRODUCCIÓN}

Los sistemas de salud del mundo no se encuentran preparados para atender el alto número de personas con enfermedades crónicas no transmisibles (ECNT). El más reciente reporte de la OMS al respecto da cuenta de un estimado de 15,2 millones de personas entre los 30 y 70 años que murieron a causa de ECNT. La región de las Américas aportó el $13 \%$ de la población a dicho estimado, mientras que en Colombia se calcula que el $74 \%$ de la mortalidad total se debe a $\mathrm{ECNT}^{1-4}$. No obstante, la enfermedad crónica no se presenta de manera aislada; por el contrario, se da como pluripatología $(P P)$. Este último término hace referencia al padecimiento de dos o más enfermedades crónicas no relacionadas entre sí. En el caso concreto de Colombia, se informa que un $1,35 \%$ de las personas padecen de múltiples enfermedades crónicas simultáneas ${ }^{2-4}$.

Las personas con PP viven con vulnerabilidad, alta dependencia y pérdida de autonomía, características que traen consigo sentimientos negativos por la frustración, la incertidumbre y el afrontamiento de su proceso de saludenfermedad ${ }^{5,6}$. Por ello se hace necesario que las personas con PP reciban cuidados específicos, continuos y que implican alta dedicación por parte del cuidador familiar ${ }^{7}$. Además, la complejidad de la PP conlleva a que sea poco reconocida dentro de ámbito sanitario, dado que la atención se encuentra fragmentada e implica la consulta a múltiples profesionales de la salud y, así mismo, un amplio número de tratamientos y exámenes que muchas veces se duplican ${ }^{8,9}$.

Esta falta de reconocimiento en la multiplicidad de morbilidades no relacionadas entre sí no solo se presenta en la práctica, sino también en la investigación dentro del contexto colombiano. Sin embargo, el creciente surgimiento de estudios internacionales relacionados con la atención integral a la persona con PP es evidencia del avance científico en torno a este fenómeno dentro de las ciencias de la salud. Por lo tanto, el objetivo de este artículo es conocer e integrar los hallazgos derivados de la producción científica disponible respecto a las experiencias y necesidades de cuidado de las personas con PP.

\section{MATERIALES Y MÉTODOS}

Se realizó una revisión integrativa descriptiva ${ }^{10}$ para responder a la pregunta “¿Cuál es el estado actual de conocimiento científico en torno a las experiencias y necesidades de las personas con pluripatología y qué vacíos investigativos se derivan de estos resultados?". La búsqueda se llevó a cabo en las bases de datos de EBSCO, Ovid, Annual Reviews, BVS, BioMed Central, ProQuest, IMAIOS, SpringerLink, Embase, Karger Journals, Latindex, PubMed, Redalyc, SciELO, ScienceDirect y Scopus, en idiomas inglés, español y portugués, y limitando entre los años 2009 y 2019. Se utilizaron las ecuaciones de búsqueda (multimorbidity, or comorbidity, or multiple chronic disease) and (nursing or health care or nursing care).

Los criterios de inclusión para esta revisión fueron artículos originales o derivados de investigación sobre el tema que incluyeran sujetos como personas con PP y/o cuidadores de estas personas, y/o profesionales de la salud que los atienden. Se excluyeron libros y tesis por su extensión, y también los artículos de reflexión y de revisión narrativa por no tener el nivel de evidencia que resulta de los procesos de investigación y que se catalogó de acuerdo con el Joanna Briggs Institute $(J B I)^{11}$. La estrategia de búsqueda y selección de artículos (Figura 1).

Se siguieron los pasos de Whittemore y $\operatorname{Kanfl}^{10}$, que incluyen la identificación del problema, la búsqueda de literatura, la evaluación y el análisis de los datos, y la presentación de los resultados. Por ello, luego de superados los dos primeros pasos, se recopilaron los artículos en el programa Microsoft Excel para el análisis de la información, donde se creó una matriz en la que se realizó una evaluación crítica de esta producción científica. Para la crítica se utilizaron los criterios de rigor en la investigación teniendo en cuenta su abordaje; para los estudios cualitativos se aplicaron los criterios de Guba ${ }^{12}$ de credibilidad, transferibilidad, dependencia y confirmabilidad, y en los estudios cuantitativos se emplearon los 
criterios de validez interna, externa, de constructo y estadística ${ }^{13}$.

Para el desarrollo de la matriz se consideraron variables como el diseño del estudio, la forma de recolección de datos, el análisis empleado, sus principales resultados, las conclusiones y los aportes a futuras investigaciones, además de la clasificación en la categoría temática correspondiente. Esta revisión tuvo en cuenta los criterios éticos establecidos respecto a las disposiciones sobre propiedad intelectual establecidas por el Congreso de Colombia en la Ley 44 de $1933^{14}$.

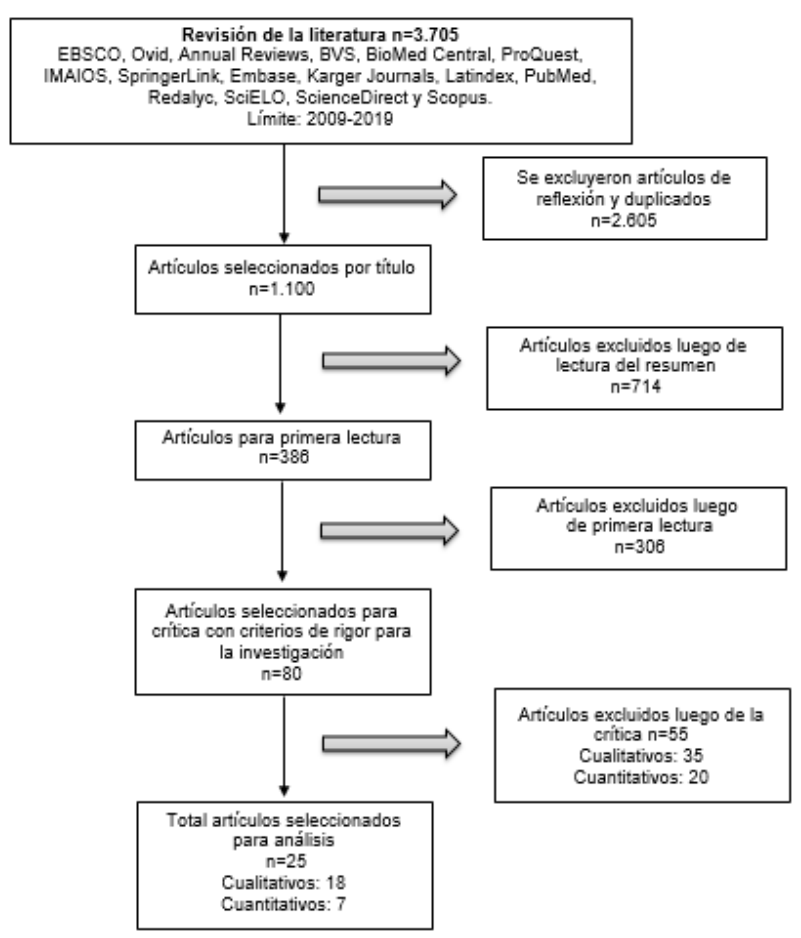

Figura 1. Diagrama de flujo de la estrategia de búsqueda y selección de artículos.

\section{RESULTADOS}

\section{Características metodológicas y temáticas de los estudios}

Se analizaron 25 artículos originales, teniendo como criterios su afinidad temática y/o metodológica. Así se obtuvieron cuatro categorías temáticas: necesidades de cuidado en la pluripatología, sentimientos ante la vivencia, barreras de atención en salud, y sistemas de apoyo. Con respecto a la distribución metodológica de los artículos luego de su integración en temática, se puede observar en la tabla 1 que el tema más abordado fue el de necesidades de cuidado en un $36 \%$. Por abordaje metodológico predominó la investigación cualitativa en un $72 \%$ del total analizado, siendo un $8 \%$ metasíntesis.

Como se ve, la tendencia en generación de conocimiento científico sobre el tema es incipiente en términos del avance en el nivel de evidencia. La tabla 2 presenta las categorías temáticas identificadas en la revisión con su nivel de evidencia predominante. Así, las necesidades de cuidado fueron el tema con mayor evidencia científica, tanto en el abordaje cualitativo como en el cuantitativo, lo que indica que ha sido el de mayor alcance investigativo. Para mayor entendimiento de esto, la $\mathrm{JBI}^{11}$ clasifica los niveles de evidencia de acuerdo a los resultados de investigación, estableciendo niveles separados para estudios que verifican efectividad, diagnóstico, pronóstico, evaluaciones económicas y significado (Tabla 2). 
Tabla 1. Distribución de 25 artículos revisados según la metodología empleada y la categoría temática.

\begin{tabular}{|c|c|c|c|c|c|c|}
\hline \multirow[b]{3}{*}{ Concepto } & \multicolumn{3}{|c|}{ Cualitativos } & \multicolumn{2}{|c|}{ Cuantitativos } & \multirow[b]{3}{*}{ Total } \\
\hline & \multirow[b]{2}{*}{$\begin{array}{l}\text { Cualitativo } \\
\text { único } \\
n(\%)\end{array}$} & \multirow[b]{2}{*}{$\begin{array}{l}\text { Revisión } \\
\text { sistemática } \\
\text { n (\%) }\end{array}$} & \multirow[b]{2}{*}{$\begin{array}{c}\text { Metasíntesis } \\
\text { n (\%) }\end{array}$} & \multirow[b]{2}{*}{$\begin{array}{c}\text { No } \\
\text { experimental } \\
n(\%)\end{array}$} & \multirow{2}{*}{$\begin{array}{c}\text { Experimental } \\
\text { Revisión } \\
\text { sistemática } \\
\mathrm{n}(\%)\end{array}$} & \\
\hline & & & & & & \\
\hline $\begin{array}{c}\text { Necesidades } \\
\text { de cuidado }\end{array}$ & $4(16)$ & 0 & $1(4)$ & $4(16)$ & $1(4)$ & $10(40)$ \\
\hline $\begin{array}{c}\text { Sentimientos } \\
\text { ante la } \\
\text { vivencia }\end{array}$ & $5(20)$ & 0 & $1(4)$ & 0 & 0 & $6(24)$ \\
\hline $\begin{array}{l}\text { Barreras en la } \\
\text { atención en } \\
\text { salud }\end{array}$ & $3(12)$ & $1(4)$ & 0 & 0 & 0 & $4(16)$ \\
\hline $\begin{array}{l}\text { Sistemas de } \\
\text { apoyo }\end{array}$ & $3(12)$ & 0 & 0 & $2(8)$ & 0 & $5(20)$ \\
\hline Total & $15(60)$ & $1(4)$ & $2(8)$ & $6(24)$ & $1(4)$ & $25(100)$ \\
\hline
\end{tabular}

Tabla 2. Resultados de niveles de evidencia por categoría temática.

\begin{tabular}{|c|c|c|}
\hline Categoría temática & Nivel de evidencia JBI & Justificación \\
\hline Necesidades de cuidado & $\begin{array}{l}\text { Nivel } 1 \text { a en efectividad } \\
\text { Nivel } 4 b \text { en efectividad } \\
\text { Nivel } 1 \text { en significado } \\
\text { Nivel } 3 \text { en significado }\end{array}$ & $\begin{array}{l}\text { Demuestra los mayores niveles de evidencia (nivel 1) } \\
\text { tanto en efectividad como en significado al } \\
\text { presentar una revisión sistemática de ECAS y una } \\
\text { metasíntesis, respectivamente. } \\
\text { También se evidencian estudios descriptivos tanto } \\
\text { de abordajes cualitativos como cuantitativos. }\end{array}$ \\
\hline $\begin{array}{l}\text { Sentimientos ante la } \\
\text { vivencia }\end{array}$ & $\begin{array}{l}\text { Nivel } 1 \text { en significado } \\
\text { Nivel } 3 \text { en significado }\end{array}$ & $\begin{array}{l}\text { Demuestra el mayor nivel de evidencia (nivel 1) en } \\
\text { significado al presentar una metasíntesis. } \\
\text { También se evidencian estudios cualitativos únicos. }\end{array}$ \\
\hline $\begin{array}{l}\text { Barreras en la atención en } \\
\text { salud }\end{array}$ & $\begin{array}{l}\text { Nivel } 2 \text { en significado } \\
\text { Nivel } 3 \text { en significado }\end{array}$ & $\begin{array}{l}\text { Presenta un nivel de evidencia en significado alto } \\
\text { por tener una revisión sistemática cualitativa. } \\
\text { Además, se evidencian estudios cualitativos únicos. }\end{array}$ \\
\hline Sistemas de apoyo & $\begin{array}{l}\text { Nivel } 4 b \text { en efectividad } \\
\text { Nivel } 3 \text { en significado }\end{array}$ & $\begin{array}{l}\text { Los estudios en este tema son de alcance descriptivo } \\
\text { tanto en los abordajes cualitativos como en los } \\
\text { cuantitativos. }\end{array}$ \\
\hline
\end{tabular}

\section{DISCUSIÓN}

A continuación, se presenta la discusión entre los hallazgos relevantes y los vacíos explícitos reportados en la literatura científica revisada, a partir de las categorías temáticas.

\section{Necesidades de cuidado}

Las necesidades de cuidado son multimodales, y abordan aspectos físicos, psicológicos, económicos, sociales, culturales y espirituales. Dentro de las de nivel físico, las personas con PP manifestaron preocupación por controlar los diferentes efectos adversos de la polifarmacia, los cuales incluyen náuseas, diarrea o estreñimiento, pérdida o aumento de peso, epigastralgia, pérdida del apetito, alteraciones del sueño, mareos, xerostomía, entre otros ${ }^{15,16}$. La polifarmacia también disminuyó su funcionalidad y autonomía, situación que, sumada a la condición propia de sus enfermedades, los hizo más dependientes ${ }^{17,18}$. 
En cuanto a la alimentación, las personas con PP requieren de una consejería nutricional acorde con sus recursos económicos que las oriente sobre formas de preparación saludable y cómo compatibilizar su dieta de PP con la del grupo familiar ${ }^{16,19,20}$. Respecto a la necesidad de autonomía, dada su situación de extrema complejidad y vulnerabilidad, es necesario hacer adecuaciones físicas y funcionales de los espacios; por ejemplo, ubicar la habitación de la persona en plantas de la casa que no requieran el uso de escaleras, instalar timbres de llamado y usar aditamentos tales como sillas de ruedas, bastones, caminadores, rampas, duchas manuales, conforme la funcionalidad de la persona ${ }^{20,21}$.

En segundo lugar, a nivel psicológico, se reportaron necesidades en torno al soporte emocional en el manejo de sentimientos negativos, así como de soporte social para la toma de decisiones, la orientación de metas y la resolución de problemas. Con frecuencia las personas con PP tienen sentimientos como ira, incertidumbre, tristeza, desesperanza y frustración ${ }^{7,15,22}$, los cuales, en general, resultan comunes ante la condición de PP dada la complejidad de esta, que compromete los mecanismos de afrontamiento y adaptación. Así pues, es indispensable que la persona y su cuidador cuenten con soporte emocional, especialmente en los momentos de crisis ${ }^{7,23}$.

De acuerdo con lo descrito, los sentimientos negativos pueden ser manejados con grupos de apoyo que favorezcan la disminución de la percepción de desesperanza, la cual es muy frecuente durante el afrontamiento de las enfermedades. La atención integral de los profesionales, además de la creación de programas que se enfoquen en la salud mental de cada uno de los pacientes, debe considerar la necesidad de la asesoría para el manejo de los medicamentos, pues muchos de estos sentimientos que se presentan son derivados de la carga del tratamiento y los efectos de esta sobre la sensación de bienestar ${ }^{21,22}$.

Considerando que el deterioro permanente, junto con la alternancia entre periodos de estabilidad y crisis, son el patrón en la vida de la persona con PP, también suele verse comprometida la capacidad de estos individuos para la toma de decisiones y la resolución de problemas; por ende, es necesario orientar metas diarias de vida, es decir, asumir cada día a la vez, con objetivos claros, viables y medibles. En este sentido, el cuidado debe buscar promover la autonomía de la persona; en particular, permitirle participar, considerando que, si esta cuenta con un estatus mental intacto, las decisiones respecto a su propia salud y vida dependerán de sí misma ${ }^{15,18}$.

A nivel económico, se identificó que el tratamiento y seguimiento de las enfermedades genera un aumento en los gastos, ya que muchas veces las personas con PP deben asumir costos que el sistema de salud no cubre a tiempo. Por ello se hace necesario proporcionar información educativa frente a los gastos que puede acarrear la PP, siendo el más frecuente el que corresponde a la realización de exámenes y medicamentos ${ }^{15,20}$. Con esto, se hace recurrente la preocupación de contar con afiliación a un sistema de salud que cubra estas necesidades, además de vincular otros sistemas como el de pensiones, para garantizar que muchas de estas personas accedan a una jubilación digna que favorezca costear sus gastos en salud ${ }^{13,17,19}$.

Frente a las necesidades a nivel social, se identifica la importancia de contar con redes de apoyo, pues la evidencia demuestra que ellas favorecen un adecuado afrontamiento ${ }^{7,20,22}$. Dentro de las principales redes se encuentran el personal de la salud, la familia, la pareja y los grupos comunitarios $^{7,18}$, los cuales han sido fundamentales para las personas durante los cambios y las crisis de las enfermedades ${ }^{18,21,23}$. Sin embargo, varios estudios describen que una de las mayores preocupaciones de las personas con PP se relacionaba con las repercusiones que podría tener su salud en el ámbito laboral porque debían dejar su trabajo, lo que les causaría inestabilidad económica ${ }^{15,19}$.

Finalmente, algunas de las necesidades a nivel espiritual se relacionan con la creación de grupos de apoyo que permitan el establecimiento de vínculos fuertes y profundos frente a las creencias propias respecto a la enfermedad, la vida y la muerte ${ }^{16-18}$. En esta medida, se reporta que grupos de la iglesia y la ayuda psicológica fueron fundamentales para 
sobrellevar el duelo por la pérdida de la salud y los sentimientos de inutilidad, ayudándoles a las personas a cultivar nuevas capacidades para adaptarse a las cargas de la enfermedad, disminuir su angustia emocional y proveer esperanza ${ }^{7,16,18}$. Para los profesionales de la salud, la literatura sugiere que un abordaje integral requiere respetar las creencias de las personas, su libertad de culto y mantenerse imparcial frente a temas controversiales como la eutanasia $20,21,23$.

\section{Sentimientos ante la vivencia}

La PP desencadena diferentes sentimientos que dependen del afrontamiento que cada persona dé a su situación de salud y que pueden ser positivos o negativos. Dentro de los sentimientos positivos, algunas investigaciones demuestran que, producto de su enfermedad, las personas experimentan la resiliencia como forma de afrontamiento ${ }^{24-26}$, sumado a que su capacidad de recuperación luego de cada crisis hacía que ganaran experiencia y confianza que les permitían controlar su proceso de salud. Es decir, se observó que hubo sujetos con PP que lograron adquirir capacidades para trazarse metas y tomar decisiones para su vida personal ${ }^{27,28}$, con lo cual ayudaban a disminuir el sentimiento de ser una carga para sus cuidadores ${ }^{28}$.

Al abordar los sentimientos negativos, la evidencia mostró múltiples sensaciones y emociones en torno al proceso de salud enfermedad de la persona con PP. Entre estos hubo muchos relacionados a la enfermedad, como la ira, la depresión, el miedo, la ansiedad, el estrés, la culpabilidad y la impotencia. Esto surge no solo por no tener control de la enfermedad o no saber cuándo se tendrá una crisis; también deriva de circunstancias sociales frente a las barreras del sistema de salud, la falta de control de las enfermedades, la polifarmacia y la poca comprensión que a veces encuentran, como pacientes, en los profesionales de la salud ${ }^{24,25,29}$.

Otros sentimientos de este tipo provienen de los problemas generados por la pérdida de independencia, dados por las limitaciones físicas derivadas de algunas patologías. Dicha dependencia acentuaba la ansiedad y la depresión ${ }^{24,26,28}$. De esta manera, cuando la persona considera que puede representar una carga para sus familiares, se presentan alteraciones emocionales caracterizadas por tristeza, disminución de la autoestima, fatiga e insomnio, lo que, sumado a sus patologías, les impide desarrollar con normalidad las actividades de la vida diaria, llevando a trastornos mentales por su afrontamiento inefectivo ante la complejidad de su salud ${ }^{28,29}$.

\section{Barreras en la atención en salud}

Las personas con PP requieren suplir tres componentes para tener satisfechas sus necesidades: la autogestión, el apoyo por parte de su cuidador y la atención de los servicios sanitarios ${ }^{30}$. Este último es el que mayores problemas les causa a las personas con PP debido a la fragmentación de la atención, la cual está ligada a la cantidad de especialistas para cada patología y el tratamiento correspondiente para cada uno de ellos. Esta falta de visión holística sobre la salud es vista como una barrera a la atención integral, lo que conlleva a la aparición de efectos adversos tras un tratamiento que no es coordinado y, en consecuencia, se genera desconfianza frente al sistema y abandono de los tratamientos ${ }^{31-33}$.

Resultado de lo anterior, otra barrera que han descrito las personas con PP está relacionada con la centralización que existe sobre las enfermedades $u$ órganos disfuncionales, dejando de lado la integridad del individuo, por lo que acuden con múltiples profesionales, ya sea para una segunda opinión o para probar un mejor tratamiento, y en algunas ocasiones se presenta contradicción entre estos. De este modo se genera incertidumbre frente a las enfermedades y las dificultades para la toma de decisiones sobre el manejo de su salud ${ }^{32,33}$. Asimismo, la carencia de la investigación frente a la PP y su manejo es otro de los obstáculos identificados ${ }^{33,34}$, pues no hay evidencia explícita sobre un abordaje holístico de estas personas y su compatibilidad con la realidad de los sistemas de salud.

Si bien las barreras en la atención involucran la acción inoportuna de los sistemas de salud, también hay barreras que son creadas por las personas con PP y que son igualmente relevantes. Dentro de estas 
se encuentran la estigmatización social frente a enfermedades como el VIH y la falta de adherencia a los tratamientos, en especial porque muchas veces no se comprende que la condición de la patología es crónica $y$, al no ver mejoría, se abandona la medicación ${ }^{32,34}$. Además, persiste una disposición inadecuada frente al autocuidado y la autogestión de la salud propia, en especial por desconocer la forma de equilibrar el manejo de las patologías ${ }^{31,32,34}$.

\section{Sistemas de apoyo}

Los sistemas de apoyo tienen un papel importante en los cambios de estilo de vida y en proceso de afrontamiento y adaptación a la condición de salud de las personas con PP y sus familias. Con frecuencia se refiere que los cuidadores principales, la familia y los profesionales de la salud son las principales redes de apoyo ${ }^{35,36}$; así, es a través de estas personas o grupos que la persona multimórbida puede expresar sus emociones y aprende estrategias para controlarlas y enfrentar el reto de vivir con ellas. De este modo, la evidencia reporta el alto valor que tiene el apoyo social efectivo en la vivencia de la PP pues, dada la alta fragilidad de la persona enferma, se requiere de interacciones significativas con actores importantes en el proceso. De hecho, se ha señalado como fundamental que haya redes de apoyo en el marco de programas como paciente experto o cuidador experto, en medio de los cuales tanto la persona enferma como su cuidador pueden interactuar con personas en situaciones de vida similares, identificarse, aconsejarse e incluso encontrar en dichas interacciones respuestas a la pregunta acerca del sentido de dicha experiencia de vida. En particular, se ha señalado que los grupos de apoyo son efectivos para disminuir los sentimientos de aislamiento, soledad y culpa ${ }^{35,36}$.

Por otra parte, algunos estudios revelan que la PP afecta las relaciones y dinámicas sociales toda vez que la persona no puede realizar actividades que generalmente hacía, aumentando sus sensaciones de soledad y aislamiento. En particular, se ha señalado que las demandas de cuidado de la persona con PP son tan altas y el cuidado es asumido con tanto compromiso que se establece una estrecha relación de cuidado entre la persona enferma y su cuidador, a tal punto que se llegan a abandonar otros roles vitales, pues se termina en una condición de interdependiencia ${ }^{35,37}$. Esta disminución en la interacción social, sin embargo, puede llegar a ocasionar sentimientos de culpa tanto en la persona enferma como en su familia y en especial en el cuidador familiar, pues la persona enferma con frecuencia se siente una carga para su entorno y, por otra parte, la familia tiende a pensar que sus cuidados no son efectivos o son erróneos. En dichas circunstancias, la persona no mejora y, por el contrario, se deteriora progresivamente ${ }^{36,38}$.

Para evitar y mitigar los efectos de la condición de PP sobre la interacción y los sistemas de apoyo social, es fundamental que los activos sociales y en salud implementen intervenciones dirigidas a esta área particular. Los grupos de apoyo en salud y basados en la comunidad han demostrado ser útiles para modular la carga de cuidado, las emociones, la toma de decisiones, la resolución de problemas y, finalmente, para aprender a vivir con una condición irreversible pero manejable. Concretamente, los grupos sociales y la religión se han considerado como fuentes de apoyo efectivo. Sin embargo, es necesario incentivar la participación e implementar horarios y metodologías flexibles, pues una de las limitaciones en la participación es la falta de tiempo y los cronogramas llenos de actividades de cuidado. Es necesario enfatizar en que la integración social favorece el descanso, la escucha, retomar energía y aprender estrategias útiles para sentirse menos abrumado ${ }^{35,37,39}$.

\section{Vacíos e implicaciones para la investigación y la práctica de los profesionales de la salud}

Tras la revisión se logró identificar factores importantes respecto a la PP, ya sea desde la posición de paciente o de profesional en salud pues, a pesar de que las percepciones no son homogéneas, revelan una tendencia hacia la fragmentación de la atención. No obstante, se evidencian a su vez herramientas que pueden ser útiles para desarrollar nuevos enfoques en la atención integral de las personas con PP, identificando las necesidades de estas frente al desarrollo de su vida con las enfermedades ${ }^{40-43}$. 
En los estudios analizados se encuentran dos posiciones frente al papel de los profesionales de la salud. La primera postura considera que es importante entender que estas personas necesitan un enfoque más integral de atención, donde la comunicación entre los profesionales facilite el abordaje y tratamiento de la PP. Esto no solo implica el manejo físico, sino la educación con respecto a las alternativas de automanejo que tienen tras la enfermedad ${ }^{4-47}$. En la segunda postura, es evidente una falta de trabajo interdisciplinario para la gestión del cuidado de la persona, considerando que no hay un enfoque de atención personalizada, empática y centrada en los pacientes, lo cual genera desconfianza $a^{40,42,46}$.

Las falencias descritas se relacionan con un factor fundamental, que es la poca investigación y el desconocimiento en la temática de PP, derivados de la fragmentación del modelo biomédico predominante en la atención ${ }^{44,47,48}$. Ante estas situaciones, los resultados de los artículos muestran algunas posibles soluciones, en especial para manejar la incertidumbre frente al diagnóstico y el tratamiento ${ }^{47-49}$. Los vacíos sugieren explorar la forma en que estas personas puedan desempeñar un papel más activo de automanejo, con el apoyo de sus cuidadores familiares ${ }^{42,43,45}$.

En términos del recorrido metodológico del área temática de la PP, puede evidenciarse que la investigación es incipiente, pues el abordaje más robusto se ha desarrollado desde la perspectiva de la gestión de la enfermedad, es decir, el estudio de condiciones crónicas individuales y no su interacción simultánea en un mismo individuo. De hecho, al clasificar las tendencias temáticas por tipo de diseño de investigación y por nivel de evidencia, se observa una amplia contribución de los estudios cualitativos en donde los sentimientos, las barreras de acceso y los sistemas de apoyo han sido descritos. De este modo queda en evidencia la necesidad de avanzar en la investigación tanto cualitativa como cuantitativa con miras a caracterizar la situación de salud de la persona con PP, además de su familia, los profesionales de salud y los sistemas de la atienden. Es imperante objetivar variables en esta población, con el fin de diseñar propuestas de intervención acordes con las particularidades de la condición $^{44,47,48}$.

Los hallazgos de esta revisión son útiles para el contexto del cuidado a la persona con PP, pues se evidencian necesidades de atención física, psicológica y social específicas, que aún no son abordadas por los profesionales de la salud y deben ser trabajadas y coordinadas por el sistema para brindar una mejor atención a esta población ${ }^{49,50}$. Por último, se destaca la importancia de crear un modelo de autogestión en el cual el paciente se comprometa con el tratamiento de sus enfermedades, teniendo en cuenta la importancia de recuperar la autonomía y disminuir su estado de vulnerabilidad $^{45,47,48,50}$.

\section{CONCLUSIÓN}

En esta revisión se encuentra que es importante brindar un cuidado integral que incluya un manejo de los problemas psicosociales y emocionales de la persona con PP, pues esta condición va más allá de las afecciones y la carga de síntomas físicos ${ }^{34,38}$. Sentirse una carga para otra persona conlleva una serie de sentimientos negativos que, aunque pueden ser comunes, deben ser abordados para favorecer el afrontamiento efectivo de la persona. A su vez, los familiares y/o el cuidador principal de estos individuos son parte fundamental de las necesidades identificadas, pues ellos también deben crear estrategias para afrontar la carga física y emocional que implica cuidar a una persona con múltiples enfermedades ${ }^{30,41}$.

También se requiere de manera importante la creación de estrategias de autogestión de la salud para que el profesional pueda no solo brindar una educación sobre las condiciones de las enfermedades, sino que proporcione herramientas de afrontamiento a estas personas y sus cuidadores, haciéndolos partícipes del tratamiento. Por ende, los sistemas de salud deben reajustar sus servicios de acuerdo a las condiciones de estos pacientes, de manera que la atención no sea fragmentada, ni centrada en una sola enfermedad. Así pues, el trabajo interprofesional surge como una de las alternativas para el cuidado de las personas con $\mathrm{PP}^{47-49}$. 
Por último, es necesario avanzar en la investigación en el área de PP, pues estos esfuerzos se han centrado en el estudio de condiciones o enfermedades crónicas y sus efectos individuales sobre la salud. Sin embargo, objetivar variables en la persona enferma, su cuidador familiar, su familia y profesionales de salud, instituciones y sistemas de salud es imperante también para cualificar la práctica del cuidado a dicha población.

\section{PROYECTO ASOCIADO}

Este artículo deriva del proyecto de investigación titulado "Efectividad de un modelo de gestión de casos para la provisión integral de servicios de salud a pacientes pluripatológicos.

\section{DECLARACIÓN DE CONFLICTOS DE INTERESES}

Los autores de este artículo declaran no tener ningún conflicto de interés en el momento de escribir y presentar los resultados de esta revisión.

\section{CONTRIBUCIÓN DE LOS AUTORES}

Primer autor: búsqueda, lectura, análisis e integración de literatura.

Segundo autor: búsqueda, lectura, análisis e integración de literatura.

Tercer autor: búsqueda, lectura, análisis e integración de literatura.

Cuarto autor: búsqueda, lectura, análisis e integración de literatura.

Quinto autor: asesoría temática y metodológica.

\section{REFERENCIAS BIBLIOGRÁFICAS}

1.Alfonso-Sierra E, Arcila-Carabalí A, Bonilla-Torres J, Latorre-Castro ML, Porras-Ramírez A, UrquijoVelásquez L. Situación de multimorbilidad en Colombia 2012-2016. BIRF-AIF Banco Mundial; $2019 . \quad$ Disponible en: http://documentos.bancomundial.org/curated/es/ 801401550612917615/pdf/134506-SPANISH-WP-
P164632-OUO-9-Multimorbilidad-en-Colombia-sinformato.pdf.

2.Hernández-Zambrano SM, Mesa-Melgarejo L, Carrillo-Algarra AJ, Castiblanco-Montañez RA, Chaparro-Diaz L, Carreño-Moreno SP, et al. Effectiveness of a case management model for the comprehensive provision of health services to multipathological people. J Adv Nurs. 2019; 75(3): 665-75. Doi: http://dx.doi.org/10.1111/jan.13892.

3.Organización Mundial de la Salud. Seguimiento de las reuniones de alto nivel de la Asamblea General de las Naciones Unidas sobre temas relacionados con salud. Prevención y control de las enfermedades no transmisibles. 72 a Asamblea Mundial de la Salud. Informe A72/19. Abril 18 de 2019 [internet] [consultado 2019 Oct 16]. Disponible en: http://apps.who.int/gb/ebwha/pdf_files/WHA72/A 72_19-sp.pdf.

4.Organización Mundial de la Salud. Noncommunicable diseases country profiles 2018 [base de datos en internet] [consultado 2019 Oct 16] Disponible en: https://www.who.int/nmh/countries/en/.

5.Gallardo Solarte K, Benavides Acosta FP, Rosales Jiménez R. Costos de la enfermedad crónica no transmisible: la realidad colombiana. Rev Cienc Salud. 2016; 14(1): 103-14. Doi: http://dx.doi.org/10.12804/revsalud14.01.2016.09.

6.Román P, Ruiz-Cantero A. La pluripatología, un fenómeno emergente y un reto para los sistemas sanitarios. Rev Clin Esp. 2017; 217(4): 229-37. Doi: https://Doi.org/10.1016/j.rce.2017.01.007.

7.Mason B, Nanton V, Epiphaniou E, Murray SA, Donaldson A, Shipman C, et al. 'My body's falling apart.' Understanding the experiences of patients with advanced multimorbidity to improve care: serial interviews with patients and carers. BMJ Support Palliat Care. 2016; 6(1): 60-5. Doi: http://dx.doi.org/10.1136/bmjspcare-2013000639.

8.Rivas-Costa G, Domínguez-Berjón $M$, AstrayMochales J, Gènova-Maleras R, Rodríguez-Laso A, 
Esteban-Vasallo M. Características epidemiológicas de la pluripatología y su influencia en la utilización de servicios sanitarios a partir de una encuesta de salud. Madrid, 2007. Rev. Esp. Salud Pública. 2009; 83(6): 835-46. Disponible en: http://scielo.isciii.es/scielo.php?script=sci_arttext\& pid=S1135-57272009000600007\&lng=es.

9.Romero M, Celis S, Marrugo R, Moreno Y, Grosso $G$, Alzate P. Caracterización de Pacientes con Múltiples Enfermedades Crónicas desde la Perspectiva del Aseguramiento en Colombia. Value Health. 2015; 18(7): 805-81. Doi: https://doi.org/10.1016/j.jval.2015.09.435.

10. Whittemore $\mathrm{R}$, Knafl K. The integrative review: updated methodology. J Adv Nurs 2005; 52(5): 54653. Doi: https://doi.org/10.1111/j.13652648.2005.03621.x.

11.Joanna Briggs Institute. New JBI Levels of Evidence. 2014.2 Available at: http://joannabriggs.org/assets/docs/approach/JBILevels-of-evidence_2014.pdf

12.Guba EG. Criteria for Assessing the Trustworthiness of Naturalistic Inquiries. ECTJ. 1981; 29: 75-91. Disponible en: https://pdfs.semanticscholar.org/8d32/23ed3c76cc 4066ec894b5aca51c4f4028b7e.pdf.

13. Hernández Sampieri R, Fernández Collado C, Baptista Lucio M. Metodología de la investigación. $6^{\circ}$ edición. México: McGraw-Hill; 2014.

14.Colombia. Congreso de la República. Ley 44 de 1993 por la cual se decretan los Derechos de autor. Diario Oficial, 40.740 (Feb. 05 1993). Disponible en: http://derechodeautor.gov.co/documents/10181/1 82597/44.pdf/7875d74e-b3ef-4a8a-8661$704823 \mathrm{~b} 871 \mathrm{~b} 5$.

15.Knowles SE, Chew-Graham C, Adeyemi I, Coupe $\mathrm{N}$, Coventry PA. Managing depression in people with multimorbidity: a qualitative evaluation of an integrated collaborative care model. BMC Fam Pract. 2015; 16(1): $32 . \quad$ Doi: https://doi.org/10.1186/s12875-015-0246-5.
16.Khan Al, Gill A, Cott C, Hans PK, Gray CS. mHealth tools for the self-management of patients with multimorbidity in primary care settings: Pilot study to explore user experience. JMIR MHealth UHealth. 2018; 6(8): e171. Doi: https://doi.org/10.2196/mhealth.8593.

17.Vermunt NP, Harmsen M, Elwyn G, Westert GP, Burgers JS, Olde Rikkert MG, et al. A three-goal model for patients with multimorbidity: A qualitative approach. Heal Expect. 2018; 21(2): 52838. Doi: https://doi.org/10.1111/hex.12647.

18.Vancampfort D, Stubbs B, Firth J, Koyanagi A. Handgrip strength, chronic physical conditions and physical multimorbidity in middle-aged and older adults in six low-and middle income countries. Eur J Intern Med. 2019; 61: 96-102. Doi: https://doi.org/10.1016/j.ejim.2018.11.007.

19.Bernabeu-Wittel M, Jadad A, Moreno-Gaviño L, Hernández-Quiles $C$, Toscano $F$, Cassani $M$, et al. Peeking through the cracks: An assessment of the prevalence, clinical characteristics and healthrelated quality of life (HRQoL) of people with polypathology in a hospital setting. Arch Gerontol Geriatr. 2010; 51(2): 185-91. Doi: https://doi.org/10.1016/j.archger.2009.10.006.

20.Siu M, Catton P, Jones J, Jadad AR. A Pilot Study Examining the Unmet Needs of Cancer Survivors Living with Polypathology. Curr Oncol. 2013; 20(3): e266-9. Doi: https://doi.org/10.3747/co.20.1307.

21.Martocchia A, Frugoni P, Indiano I, Tafaro L, Comite F, Amici A, et al. Screening of frailty in elderly patients with disability by the means of MariglianoCacciafesta polypathology scale (MCPS) and Canadian Study of Health and Aging (CSHA) scales. Arch Gerontol Geriatr. 2013; 56(2): 339-42. Doi: https://doi.org/10.1016/j.archger.2012.11.004.

22. Cottrell E, Yardley S. Lived experiences of multimorbidity: An interpretative meta-synthesis of patients', general practitioners' and trainees' perceptions. Chronic Illn. 2015; 11(4): 279-303. Doi: https://doi.org/10.1177/1742395315574764. 
23.Baker JM, Grant RW, Gopalan A. A systematic review of care management interventions targeting multimorbidity and high care utilization. BMC Health Serv Res. 2018; 18(1): 65. Doi: https://doi.org/10.1186/s12913-018-2881-8.

24. Hays R, Daker-White G, Esmail A, Barlow W, Minor B, Brown B, et al. Threats to patient safety in primary care reported by older people with multimorbidity: baseline findings from a longitudinal qualitative study and implications for intervention. BMC Health Serv Res. 2017; 17(1): 754. Doi: https://doi.org/10.1186/s12913-017-2727-9.

25.Coventry PA, Small N, Panagioti M, Adeyemi I, Bee P. Living with complexity; marshalling resources: a systematic review and qualitative metasynthesis of lived experience of mental and physical multimorbidity. BMC Fam Pract. 2015; 16(1): 171. Doi: https://doi.org/10.1186/s12875-015-0345-3.

26.Janke EA, Ramirez ML, Haltzman B, Fritz M, Kozak AT. Patient's experience with comorbidity management in primary care: a qualitative study of comorbid pain and obesity. Prim Health Care Res Dev. 2016; 17(1): 33-41. Doi: https://doi.org/10.1017/S1463423615000171.

27.Eckerblad J, Theander K, Ekdahl A, Jaarsma T, Hellstrom I. To adjust and endure: a qualitative study of symptom burden in older people with multimorbidity. Appl Nurs Res. 2015; 28(4): 322-7. Doi: https://doi.org/10.1016/j.apnr.2015.03.008.

28.Jarling A, Rydström I, Ernsth-Bravell M, Nyström $M$, Dalheim-Englund $A$. Becoming a guest in your own home: Home care in Sweden from the perspective of older people with multimorbidities. Int J Older People Nurs. 2018; 13(3): e12194. Doi: https://doi.org/10.1111/opn.12194.

29.Leijten FRM, Hoedemakers M, Struckmann V, Kraus $M$, Cheraghi-Sohi $S$, Zemplényi $A$, et al. Defining good health and care from the perspective of persons with multimorbidity: results from a qualitative study of focus groups in eight European countries. BMJ Open. 2018; 8(8): e021072. Doi: https://doi.org/10.1136/bmjopen-2017-021072.
30.Bernabeu-Wittel $M$, Alonso-Coello P, RicoBlázquez M, del Campo RR, Gómez SS, Vales EC. Desarrollo de guías de práctica clínica en pacientes con comorbilidad y pluripatología. Atención Primaria. 2014; 46(7): 385-92. Doi: https://doi.org/10.1016/j.aprim.2013.11.013.

31.Rosbach M, Andersen JS. Patient-experienced burden of treatment in patients with multimorbidity-A systematic review of qualitative data. PLoS One. 2017; 12(6): e0179916. Doi: https://doi.org/10.1371/journal.pone.0179916.

32.Van Merode T, Van De Ven K, Van Den Akker M. Patients with Multimorbidity and Their Treatment Burden in Different Daily Life Domains: A Qualitative Study in Primary Care in the Netherlands and Belgium. J Comorbidity. 2018; 8(1): 9-15. Doi: https://doi.org/10.15256/joc.2018.8.119.

33.Stokes T, Tumilty E, Doolan-Noble F, Gauld R. Multimorbidity, clinical decision making and health care delivery in New Zealand Primary care: a qualitative study. BMC Fam Pract. 2017; 18(1): 51. Doi: https://doi.org/10.1186/s12875-017-0622-4.

34.Daker-White G, Hays R, Blakeman T, Croke $S$, Brown B, Esmail A, et al. Safety work and risk management as burdens of treatment in primary care: insights from a focused ethnographic study of patients with multimorbidity. BMC Fam Pract. 2018; 19(1): 155. Doi: https://doi.org/10.1186/s12875018-0844-0.

35.Chang AY, Gómez-Olivé FX, Manne-Goehler J, Wade AN, Tollman S, Gaziano TA, et al. Multimorbidity and care for hypertension, diabetes and HIV among older adults in rural South Africa. Bull World Health Organ. 2019; 97(1): 10. Doi: https://doi.org/10.2471/BLT.18.217000.

36.Rijken M, Hujala A, van Ginneken E, Melchiorre MG, Groenewegen P, Schellevis F. Managing multimorbidity: Profiles of integrated care approaches targeting people with multiple chronic conditions in Europe. Health Policy. 2018; 122(1): 44-52.

Doi: https://doi.org/10.1016/j.healthpol.2017.10.002. 
37. Matima R, Murphy K, Levitt NS, BeLue R, Oni T. A qualitative study on the experiences and perspectives of public sector patients in Cape Town in managing the workload of demands of HIV and type 2 diabetes multimorbidity. PLoS One. 2018; 13(3): $\quad$ e0194191. Doi: https://doi.org/10.1371/journal.pone.0194191.

38. Coventry PA, Fisher L, Kenning C, Bee P, Bower P. Capacity, responsibility, and motivation: a critical qualitative evaluation of patient and practitioner views about barriers to self-management in people with multimorbidity. BMC Health Serv Res. 2014; 14(1): 536. Doi: https://doi.org/10.1186/s12913014-0536-y.

39.Slomka J, Prince-Paul M, Webel A, Daly BJ. Multimorbidity With HIV: Views of CommunityBased People Living With HIV and Other Chronic Conditions. J Assoc Nurses AIDS Care. 2017; 28(4): 603-11.

Doi:

https://doi.org/10.1016/j.jana.2017.04.003.

40. Harrison C, Henderson J, Miller G, Britt H. The prevalence of complex multimorbidity in Australia. Aust N Z J Public Health. 2016; 40(3): 239-44. Doi: https://doi.org/10.1111/1753-6405.12509.

41.Boehmer KR, Guerton NM, Soyring J, Hargraves I, Dick S, Montori VM. Capacity coaching: a new strategy for coaching patients living with multimorbidity and organizing their care. Mayo Clin. Proc. 2019; 94(2): 278-86. Doi: https://doi.org/10.1016/j.mayocp.2018.08.021.

42.Poitras $M-E$, Maltais $M-E$, Bestard-Denommé $L$, Stewart $M$, Fortin $M$. What are the effective elements in patient-centered and multimorbidity care? A scoping review. BMC Health Serv Res. 2018; 18(1): 446. Doi: https://doi.org/10.1186/s12913018-3213-8.

43. Díez-Manglano J. La pluripatología, un reto para los sistemas sanitarios. Rev Clín Esp. 2017; 217(4): 207.

Doi:

https://doi.org/10.1016/j.rce.2017.01.002.

44.Ledón-Llanes L. Enfermedades crónicas y vida cotidiana. Rev Cub Salud Pública. 2011; 37(4): 488-
$499 . \quad$ Disponible en: http://scielo.sld.cu/scielo.php?script=sci_arttext\&p id=S0864-34662011000400013

45.Bandera López M, Escaño Cardona V, Caballero León M. Relato biográfico expresado desde la narrativa de una cuidadora joven a cargo un paciente con pluripatologías. Enferm Docente. 2016; (107): 43-46. Disponible en: http://www.index-

f.com/edocente/107pdf/10742.pdf.

46.Organización de las Naciones Unidas [Internet]. Protocolo facultativo de la Convención sobre los derechos de las personas con discapacidad. 2007. Disponible

en: https://www.ohchr.org/SP/Professionallnterest/Pa ges/OptionalProtocolRightsPersonsWithDisabilities. aspx.

47.Pérez Giménez G. Impacto de un programa de gestión de casos a pacientes crónicos complejos. [Tesis doctoral de Enfermería]. Barcelona, España: Universitat de Barcelona; 2017. Disponible en: http://diposit.ub.edu/dspace/bitstream/2445/1181 70/1/GPG_TESIS.pdf.

48.Nuño-Solinís R. Desarrollo e implementación de la Estrategia de Cronicidad del País Vasco: lecciones aprendidas. Gac Sanit. 2016; 30(1): 106-10 Doi: http://dx.doi.org/10.1016/j.gaceta.2016.03.011.

49.Sociedad Española de Medicina Interna (SEMI), Sociedad Española de Medicina de Familia y Comunitaria (semFYC), Federación de Asociaciones de Enfermería Comunitaria y Atención Primaria (FAECAP). Desarrollo de guías de práctica clínica en pacientes con comorbilidad y pluripatología. Madrid, España: Multimédica Proyectos; 2013. Disponible en: https://www.semfyc.es/wpcontent/uploads/2016/05/DesarrolloGuiasPluripat ologia.pdf.

50.Carreño-Moreno S, Chaparro-Díaz L. Calidad de vida de los cuidadores de personas con enfermedad crónica. Aquichan. 2016; 16(4): 447-61. Doi: https://doi.org/10.5294/aqui.2016.16.4.4 